\title{
CHANNEL FLOW OF A VISCOUS FLUID IN THE BOUNDARY LAYER
}

\author{
BY \\ C. Y. CHAN AND P. C. KONG* \\ University of Southwestern Louisiana, Lafayette, Louisiana
}

1. Introduction. Let $u$ be the temperature, $\psi$ be a stream function, and $x$ and $t$ be, respectively, the coordinates perpendicular and parallel to the channel walls. Ockendon [5] described the flow of a viscous fluid in the boundary layer by the following initialboundary value problem:

$$
\begin{aligned}
& \psi_{x x}=e^{u}, \\
& u_{x x}+\psi_{x} u_{t}-\psi_{t} u_{x}=-e^{u}, \\
& u(x, 0)=0, \quad \psi(x, 0)=\frac{1}{2} x^{2}\left(1-\frac{1}{3} \beta^{-1 / 2} x\right) \quad \text { for } x>0, \\
& u(0, t)=0, \quad \psi(0, t)=0, \quad \psi_{x}(0, t)=0=\psi_{t}(0, t) \quad \text { for } t>0,
\end{aligned}
$$

where $\beta$, called the Nahme-Griffith number, is assumed to be large. For some positive constant $a\left(\leq 2 \beta^{1 / 2}\right)$, let $\Omega=(0, a) \times(0, T)$, and $\partial \Omega$ be the parabolic boundary $([0, a] \times$ $\{0\}) \cup(\{0, a\} \times(0, T))$ of $\Omega$, where $T \leq \infty$. Ockendon found that as $x \rightarrow \infty, u \sim t / x$ and $\psi \sim x^{2} / 2$. Since $\beta$ is very large, $u \rightarrow 0$ as $x \rightarrow 2 \beta^{1 / 2}$. Thus, the model of the channel flow of a fluid with temperature-dependent viscosity in the boundary layer is simplified to the following degenerate parabolic problem (cf. Lacey [4], Stuart and Floater [6], and Floater [3]),

$$
u_{x x}-x u_{t}=-e^{u} \quad \text { in } \Omega, \quad u=0 \text { on } \partial \Omega,
$$

where $u$ may blow up at some finite $T$.

Recently, Floater [3] studied the problem (1.1). He approximated the forcing term $e^{u}$ by $u^{p}$. Instead of zero initial temperature, he considered the following problem:

$$
\begin{gathered}
u_{x x}-x u_{t}=-u^{p} \quad \text { in }(0,1) \times(0, \infty), \\
u(x, 0)=u_{0}(x) \geq 0 \quad \text { for } x \in[0,1], \quad u(0, t)=0=u(1, t) \quad \text { for } t>0,
\end{gathered}
$$

where $p>1$ and $u_{0}$ is assumed to be in $C^{1}([0,1])$ with $u_{0}(0)=0=u_{0}(1)$. He showed that if $1<p \leq 2$ and $u_{0}$ is concave, then $u$ blows up at the boundary $x=0$. Numerical

Received January 26, 1994.

1991 Mathematics Subject Classification. Primary 35K65, 35K60, 35K57; Secondary 76D10.

* Current address: Carson Newman College, Jefferson City, Tennessee 
evidence by Stuart and Floater [6] indicates that blow-up occurs away from the boundary when $p>2$.

Let $q$ be any real number, and

$$
L \equiv \frac{\partial^{2}}{\partial x^{2}}-x^{q} \frac{\partial}{\partial t}
$$

When $q=0, L$ is the ordinary heat operator. When $q \neq 0$, the coefficient of $\partial / \partial t$ in $L$ becomes zero or infinity at $x=0$, and we say that $L$ is a degenerate parabolic operator. Floater [3] generalized the problem (1.2) by replacing the equation with

$$
L u=-u^{p} \quad \text { in }(0,1) \times(0, \infty)
$$

for any $q>0$, and he found that blow-up at the boundary $x=0$ can occur when $1<p \leq q+1$.

To study the problem (1.1), we use a different approach. Let

$$
h_{r}(u)=\left(1-\frac{u}{r}\right)^{-r} .
$$

We note that for each fixed $r(>0), e^{u}<h_{r}(u)$ for $u \in(0, r)$. Also, $\lim _{r \rightarrow \infty} h_{r}(u)=e^{u}$. Let us consider the following problem:

$$
L u=-h_{r}(u) \text { in } \Omega, \quad u=0 \quad \text { on } \partial \Omega .
$$

We see that the problem (1.1) is the limiting case of the problem (1.3) with $q=1$ as $r$ tends to infinity. We also note that for each fixed $r, h_{r}(0)=1, h_{r}^{\prime}>0, h_{r}^{\prime \prime}>0$, $\lim _{u \rightarrow r^{-}} h_{r}(u)=\infty$, and $\int_{0}^{r} h_{r}(u) d u=\infty$ for $r>1$. This motivates us to study the more general degenerate parabolic quenching problem,

$$
L u=-f(u) \quad \text { in } \Omega, \quad u=0 \quad \text { on } \partial \Omega
$$

for $q \neq 0$, where $f \in C^{2}([0, c))$ for some positive constant $c$ such that $f(0)>0, f^{\prime}>0$, $f^{\prime \prime} \geq 0, \lim _{u \rightarrow c^{-}} f(u)=\infty$, and $\int_{0}^{c} f(u) d u=\infty$. By quenching phenomena, we mean the blow-up of $u_{t}$ at some finite $T$ and existence of a unique critical length $a^{*}$ (which is the length such that for $a<a^{*}, u$ exists for all $t(>0)$, and for $a>a^{*}, u$ reaches $c$ somewhere at some finite $T$ (and $u_{t}$ blows up there)).

Chan and Kong [1] showed that the problem (1.4) has a unique classical solution $u$ such that $u$ and $u_{t}$ are positive in $\Omega$, and there exists a unique critical length $a^{*}$, which is the same as that for the case $q=0$; if $\int_{0}^{c} f(u) d u=M$ for some positive constant $M$ and $a>a^{*}$, then $u$ reaches $c$ somewhere in

$$
\begin{array}{ll}
{\left[c^{2} /(2 M a), a / 2\right]} & \text { for } q>0, \\
{\left[a / 2, a-c^{2} /(2 M a)\right]} & \text { for } q<0,
\end{array}
$$

and $\lim _{(x, t) \rightarrow\left(x_{0}, T\right)} u_{t}(x, t)=\infty$ if $\lim _{(x, t) \rightarrow\left(x_{0}, T\right)} u(x, t)=c$ for some finite $T$.

In Sec. 2, we show that without assuming $\int_{0}^{c} f(u) d u=M$, if $a>a^{*}$, then $u$ reaches $c$ somewhere in

$$
\begin{aligned}
& {[0, a / 2] \text { for } q>0} \\
& {[a / 2, a] \text { for } q<0}
\end{aligned}
$$

if $\int_{0}^{c} f(u) d u=\infty$, then $u_{t}$ becomes unbounded somewhere when $u$ reaches $c$ at some finite $T$. 
2. Blow-up of $u_{t}$. The following result is given by Chan and Kong [1].

TheOrem 1. The problem (1.4) has a unique classical solution $u$ such that $u$ and $u_{t}$ are positive in $\Omega$.

We remark that if quenching does not occur, then Theorem 1 gives the existence result for all $t>0$.

Let $\omega_{-}=(0, a / 2) \times(0, T)$ and $\omega_{+}=(a / 2, a) \times(0, T)$. Since $u_{t}>0$ in $\Omega$, we use the strong maximum principle to prove the following lemma.

LEMma 2. For the problem (1.4), (i) if $q>0$, then $u_{x}(x, t)<0$ in $\omega_{+}$; (ii) if $q<0$, then $u_{x}(x, t)>0$ in $\omega$

Proof. (i) Let $\alpha$ be a positive number less than or equal to $a / 2, \Omega_{\alpha}=(a-2 \alpha$, $a-\alpha) \times(0, T)$, and $y(x, t)=u(x, t)-u(2 a-2 \alpha-x, t)$. For $x \in(a-2 \alpha, a-\alpha)$, we have $2 a-2 \alpha-x>x$. It follows from $q>0$ and $u_{t}>0$ in $\Omega$ that

$$
\left(L+f^{\prime}(\xi)\right) y<0 \quad \text { in } \Omega_{\alpha},
$$

where $\xi(x, t)$ is between $u(x, t)$ and $u(2 a-2 \alpha-x, t)$. Since $u=0$ on the parabolic boundary $\partial \Omega$, we have $y(a-2 \alpha, t) \geq 0$, and $y(x, 0)=0$. Also, $y(a-\alpha, t)=0$. Hence, $y>0$ in $\Omega_{\alpha}$ by the strong maximum principle. In particular, for any $\epsilon$ such that $0<\epsilon<\alpha$,

$$
u(a-\alpha-\epsilon, t)>u(a-\alpha+\epsilon, t) .
$$

Thus, $u_{x}(a-\alpha, t) \leq 0$. From this,

$$
u_{x}(x, t) \leq 0 \text { for } x \in\left[\frac{a}{2}, a\right)
$$

We note that

$$
\left(u_{x}\right)_{x x}-x^{q}\left(u_{x}\right)_{t}+f^{\prime}(u) u_{x}=q x^{q-1} u_{t} .
$$

Since $q>0$ and $u_{t}>0$, we have

$$
\left(L+f^{\prime}(u)\right) u_{x}>0 \text { in } \omega_{+} .
$$

If $u_{x}$ attains its maximum 0 at some point (say, $\left.\left(x^{*}, \sigma\right)\right)$ in $\omega_{+}$, then by the strong maximum principle, $u_{x}(x, t) \equiv 0$ in $(a / 2, a) \times(0, \sigma]$, and hence $\left(L+f^{\prime}(u)\right) u_{x}=0$ there. This contradiction shows that $u_{x}(x, t)<0$ in $\omega_{+}$.

(ii) The proof is similar to that for (i).

From the proofs of Theorems 5 and 6 of Chan and Kong [1], the problem (1.4) has a unique critical length $a^{*}$. When $a>a^{*}$, it follows from Lemma 2 that $u$ reaches $c$ somewhere in $[0, a / 2]$ for $q>0$, and in $[a / 2, a]$ for $q<0$.

We modify the idea in the proof of Theorem 1 of Chan and Kwong [2] to prove the following result.

Theorem 3. For the problem (1.4), suppose $\int_{0}^{c} f(u) d u=\infty$. As $t \rightarrow T^{-}$, if $u(x, t) \rightarrow c^{-}$ at some $x \in[0, a]$, then $u_{t}(x, t) \rightarrow \infty$ at (at least) one of such $x$.

Proof. Suppose $u_{t}$ is bounded on $\bar{\Omega}$. Let us choose a positive number $K$ such that $u_{t}<K$ on $\bar{\Omega}$ and $K>8 c / a^{2}$. 
We first consider the case $q>0$. Since $\lim _{u \rightarrow c^{-}} f(u)=\infty$, there exists a constant $\beta_{1}(>3 c / 4)$ such that

$$
f(u)>\left(1+a^{q}\right) K \quad \text { for } u \in\left[\beta_{1}, c\right) .
$$

It follows from Lemma $2(\mathrm{i})$ and $\int_{0}^{c} f(u) d u=\infty$ that we can choose a constant $\tau_{1}$ close to $T$ such that $u\left(x, \tau_{1}\right)$ attains a local maximum at some point $x^{*} \in(0, a / 2]$ with the properties $u\left(x^{*}, \tau_{1}\right)>\beta_{1}$ and

$$
\int_{\beta_{1}}^{u\left(x^{*}, \tau_{1}\right)} f(s) d s>\frac{1+a^{q}}{2}\left(\frac{4 \beta_{1}}{a}+\frac{K a^{1+q}}{8}\right)^{2} .
$$

When $u>\beta_{1}, u_{x x}<a^{q} K-f(u)$. By (2.1), $u_{x x}<-K<0$. Hence, there exists $x_{1}$ such that $x^{*}<x_{1}<a, u\left(x_{1}, \tau_{1}\right)=\beta_{1}$, and $u_{x}\left(x, \tau_{1}\right)<0$ in $\left(x^{*}, x_{1}\right)$. For $x \in\left(x^{*}, x_{1}\right)$, we have $u\left(x, \tau_{1}\right)>\beta_{1}$. It follows from $q>0, u_{t}<K$, and (2.1) that

$$
\begin{aligned}
u_{x x}\left(x, \tau_{1}\right) & <a^{q} K-f\left(u\left(x, \tau_{1}\right)\right) \\
& <-\frac{1}{1+a^{q}} f\left(u\left(x, \tau_{1}\right)\right) .
\end{aligned}
$$

Since $u_{x}\left(x, \tau_{1}\right)<0$ in $\left(x^{*}, x_{1}\right)$, we have

$$
u_{x}\left(x, \tau_{1}\right) u_{x x}\left(x, \tau_{1}\right)>-\frac{1}{1+a^{q}} f\left(u\left(x, \tau_{1}\right)\right) u_{x}\left(x, \tau_{1}\right)
$$

Upon integrating from $x^{*}$ to $x_{1}$, we obtain

$$
\frac{u_{x}^{2}\left(x_{1}, \tau_{1}\right)}{2}>\frac{1}{1+a^{q}} \int_{\beta_{1}}^{u\left(x^{*}, \tau_{1}\right)} f(s) d s .
$$

By (2.2),

$$
u_{x}^{2}\left(x_{1}, \tau_{1}\right)>\left(\frac{4 \beta_{1}}{a}+\frac{K a^{1+q}}{8}\right)^{2} .
$$

Since $u_{x}\left(x_{1}, \tau_{1}\right) \leq 0$, we have

$$
u_{x}\left(x_{1}, \tau_{1}\right)<-\left(\frac{4 \beta_{1}}{a}+\frac{K a^{1+q}}{8}\right) .
$$

We claim that $a-x_{1}>a / 4$. For $x \in\left(x^{*}, x_{1}\right)$, we have $u\left(x, \tau_{1}\right)>\beta_{1}$. Thus, $u_{x x}\left(x, \tau_{1}\right)<$ $-K$, and we have

$$
\int_{x^{*}}^{x_{1}} \int_{x^{*}}^{x} u_{\xi \xi}\left(\xi, \tau_{1}\right) d \xi d x<-K \int_{x^{*}}^{x_{1}} \int_{x^{*}}^{x} d \xi d x
$$

which gives

$$
\beta_{1}-u\left(x^{*}, \tau_{1}\right)<-\frac{K}{2}\left(x_{1}-x^{*}\right)^{2} .
$$

Since $u<c, \beta_{1}>3 c / 4$, and $K>8 c / a^{2}$, we have $x_{1}-x^{*}<a / 4$. It follows from $x^{*} \in(0, a / 2]$ that $a-x_{1}=a-x^{*}-\left(x_{1}-x^{*}\right)>a / 4$. 
Since $u_{t}<K$ and $f(u)>0$, we have $u_{x x}\left(x, \tau_{1}\right)<a^{q} K$. By $(2.3)$,

$$
\int_{x_{1}}^{x_{1}+\frac{a}{4}} \int_{x_{1}}^{x} u_{\xi \xi}\left(\xi, \tau_{1}\right) d \xi d x<a^{q} K \int_{x_{1}}^{x_{1}+\frac{a}{4}} \int_{x_{1}}^{x} d \xi d x
$$

gives

$$
u\left(x_{1}+\frac{a}{4}, \tau_{1}\right)<\beta_{1}-\left(\frac{4 \beta_{1}}{a}+\frac{K a^{1+q}}{8}\right)\left(\frac{a}{4}\right)+\frac{K a^{q}}{2}\left(\frac{a}{4}\right)^{2}=0 .
$$

This contradicts $u>0$ in $\Omega$.

Now, we consider the case $q<0$. By Lemma 2(ii), $u(x, t)<c$ for $x \in(0, a / 2)$. Thus, there exists a positive constant $b(<c)$ such that $u(a / 8, t) \leq b$ for $t>0$. Since $\lim _{u \rightarrow c^{-}} f(u)=\infty$, there exists a constant $\beta_{2}$ such that $\beta_{2}>\max \{3 c / 4, b\}$ and

$$
f(u)>\left[1+\left(\frac{a}{8}\right)^{q}\right] K \quad \text { for } u \in\left[\beta_{2}, c\right) .
$$

It follows from Lemma 2 (ii) and $\int_{0}^{c} f(u) d u=\infty$ that we can choose a constant $\tau_{2}$ close to $T$ such that $u\left(x, \tau_{2}\right)$ attains a local maximum at some point $x_{*} \in[a / 2, a)$ with the properties $u\left(x_{*}, \tau_{2}\right)>\beta_{2}$ and

$$
\int_{\beta_{2}}^{u\left(x_{*}, \tau_{2}\right)} f(s) d s>\frac{1}{2}\left[1+\left(\frac{a}{8}\right)^{q}\right]\left[\frac{8 \beta_{2}}{a}+\frac{K}{2}\left(\frac{a}{8}\right)^{1+q}\right]^{2} .
$$

When $u>\beta_{2}$, we have $x>a / 8$. It follows from $q<0$ and (2.4) that

$$
u_{x x}<\left(\frac{a}{8}\right)^{q} K-f(u)<-K<0 .
$$

Hence, there exists $x_{2}$ such that $a / 8<x_{2}<x_{*}, u\left(x_{2}, \tau_{2}\right)=\beta_{2}$, and $u_{x}\left(x, \tau_{2}\right)>0$ in $\left(x_{2}, x_{*}\right)$. Then, it follows from $u_{t}<K$ and (2.4) that for $x \in\left(x_{2}, x_{*}\right)$,

$$
\begin{aligned}
u_{x x}\left(x, \tau_{2}\right) & <\left(\frac{a}{8}\right)^{q} K-f\left(u\left(x, \tau_{2}\right)\right) \\
& <-\frac{1}{1+(a / 8)^{q}} f\left(u\left(x, \tau_{2}\right)\right) .
\end{aligned}
$$

Since $u_{x}\left(x, \tau_{2}\right)>0$ in $\left(x_{2}, x_{*}\right)$, we have

$$
u_{x}\left(x, \tau_{2}\right) u_{x x}\left(x, \tau_{2}\right)<-\frac{1}{1+(a / 8)^{q}} f\left(u\left(x, \tau_{2}\right)\right) u_{x}\left(x, \tau_{2}\right) .
$$

Integrating from $x_{2}$ to $x_{*}$, we have

$$
-\frac{u_{x}^{2}\left(x_{2}, \tau_{2}\right)}{2}<-\frac{1}{1+(a / 8)^{q}} \int_{\beta_{2}}^{u\left(x_{*}, \tau_{2}\right)} f(s) d s .
$$

By (2.5),

$$
u_{x}^{2}\left(x_{2}, \tau_{2}\right)>\left[\frac{8 \beta_{2}}{a}+\frac{K}{2}\left(\frac{a}{8}\right)^{1+q}\right]^{2}
$$


Since $u_{x}\left(x_{2}, \tau_{2}\right) \geq 0$, we have

$$
u_{x}\left(x_{2}, \tau_{2}\right)>\frac{8 \beta_{2}}{a}+\frac{K}{2}\left(\frac{a}{8}\right)^{1+q} .
$$

We claim that $x_{2}>a / 4$. For $x \in\left(x_{2}, x_{*}\right)$, we have $u\left(x, \tau_{2}\right)>\beta_{2}$. By (2.4), $u_{x x}\left(x, \tau_{2}\right)<-K$. Then,

$$
\int_{x_{2}}^{x_{*}} \int_{x}^{x_{*}} u_{\xi \xi}\left(\xi, \tau_{2}\right) d \xi d x<-K \int_{x_{2}}^{x_{*}} \int_{x}^{x_{*}} d \xi d x
$$

which gives

$$
\beta_{2}-u\left(x_{*}, \tau_{2}\right)<-\frac{K}{2}\left(x_{*}-x_{2}\right)^{2}
$$

Since $u<c, \beta_{2}>3 c / 4$, and $K>8 c / a^{2}$, we have $x_{*}-x_{2}<a / 4$. It follows from $x_{*} \in[a / 2, a)$ that $x_{2}=x_{*}-\left(x_{*}-x_{2}\right)>a / 4$.

Since $u_{t}<K$ and $f(u)>0$, we have $u_{x x}\left(x, \tau_{2}\right)<(a / 8)^{q} K$ for $x>a / 8$. Thus,

$$
\int_{x_{2}-\frac{a}{8}}^{x_{2}} \int_{x}^{x_{2}} u_{\xi \xi}\left(\xi, \tau_{2}\right) d \xi d x<\left(\frac{a}{8}\right)^{q} K \int_{x_{2}-\frac{a}{8}}^{x_{2}} \int_{x}^{x_{2}} d \xi d x
$$

gives

$$
u\left(x_{2}-\frac{a}{8}, \tau_{2}\right)<\beta_{2}-\frac{a}{8}\left[u_{x}\left(x_{2}, \tau_{2}\right)-\frac{K}{2}\left(\frac{a}{8}\right)^{1+q}\right] .
$$

By (2.6), $u\left(x_{2}-a / 8, \tau_{2}\right)<0$. This contradicts $u>0$ in $\Omega$. Hence, the theorem is proved.

\section{References}

[1] C. Y. Chan and P. C. Kong, Quenching for degenerate semilinear parabolic equations, Appl. Anal. 54, 17-25 (1994)

[2] C. Y. Chan and M. K. Kwong, Quenching phenomena for singular nonlinear parabolic equations, Nonlinear Anal. 12, 1377-1383 (1988)

[3] M. S. Floater, Blow-up at the boundary for degenerate semilinear parabolic equations, Arch. Rational Mech. Anal. 114, 57.77 (1991)

[4] A. A. Lacey, The form of blow-up for nonlinear parabolic equations, Proc. Roy. Soc. Edinburgh 98A, 183-202 (1984)

[5] H. Ockendon, Channel flow with temperature-dependent viscosity and internal viscous dissipation, J. Fluid Mech. 93, $737 \cdot 746$ (1979)

[6] A. M. Stuart and M. S. Floater, On the computation of blow-up, European J. Appl. Math. 1, 41-71 (1990) 